\title{
SOME RESULTS ON THE ASYMPTOTIC BEHAVIOR OF NONOSCILLATORY SOLUTIONS OF DIFFERENTIAL EQUATIONS WITH DEVIATING ARGUMENTS
}

\author{
CH. G. PHILOS, Y. G. SFICAS and V. A. STAIKOS
}

(Received 2 June 1977; revised 6 February 1981)

Communicated by H. Lausch \& G. R. Morris

\begin{abstract}
This paper deals with some asymptotic properties of nonoscillatory solutions of a class of $n$-th order $(n>1)$ differential equations with deviating arguments involving the so called $n$-th order $r$-derivative of the unknown function $x$ defined by

$$
D_{r}^{(n)} x=r_{n}\left(r_{n-1}\left(r_{n-2}\left(\cdots\left(r_{1}\left(r_{0} x\right)^{\prime}\right)^{\prime} \cdots\right)^{\prime}\right)^{\prime}\right)^{\prime},
$$

where $r_{i}(i=0,1, \ldots, n)$ are positive continuous functions on $\left[t_{0}, \infty\right)$. The fundamental purpose of this paper is to find for any integer $m, 0 \leqslant m \leqslant n-1$, a necessary and sufficient condition (depending on $m$ ) in order that there exists at least one (nonoscillatory) solution $x$ so that the $\lim _{t \rightarrow \infty}\left(D_{r}^{(m)} x\right)(t)$ exists in $\mathbf{R}-\{0\}$. The results obtained extend some recent ones due to Philos (1978a) and also they prove, in a general setting, the validity of a conjecture made by Kusano and Onose (1975).
\end{abstract}

1980 Mathematics subject classification (Amer. Math. Soc.): 34 C 10, 34 K 25.

\section{Introduction}

Let $r_{i}(i=0,1, \ldots, n)$ be positive continuous functions on the interval $\left[t_{0}, \infty\right)$. For a real valued function $h$ on $[T, \infty), T \geqslant t_{0}$, and any $\mu=0,1, \ldots, n$ we define the $\mu$-th $r$-derivative of $h$ by the formula

$$
D_{r}^{(\mu)} h=r_{\mu}\left(r_{\mu-1}\left(\cdots\left(r_{1}\left(r_{0} h\right)^{\prime}\right)^{\prime} \cdots\right)^{\prime}\right)^{\prime} .
$$

During the preparation of this paper the first author was supported by the National Hellenic Research Foundation.

(c) Copyright Australian Mathematical Society 1982 
Then we obviously have

$$
D_{r}^{(0)} h=r_{0} h \quad \text { and } \quad D_{r}^{(i)} h=r_{i}\left(D_{r}^{(i-1)} h\right)^{\prime} \quad(i=1,2, \ldots, n) .
$$

If $D_{r}^{(n)} h$ is defined on $[T, \infty)$, then the function $h$ is called $n$-times $r$-differentiable and if, in addition, $D_{r}^{(n)} h$ is continuous, $h$ is called $n$-times continuously $r$ differentiable.

Now, we consider the $n$-th order $(n>1)$ differential equation with deviating arguments of the form

$$
\left(D_{r}^{(n)} x\right)(t)+\delta F(t ; x\langle g(t)\rangle)=b(t),
$$

where $\delta= \pm 1$ and

$$
x\langle g(t)\rangle=\left(x\left[g_{1}(t)\right], x\left[g_{2}(t)\right], \ldots, x\left[g_{N}(t)\right]\right), \quad g=\left(g_{1}, g_{2}, \ldots, g_{N}\right) .
$$

Obviously, there is no loss of generality for our purposes to suppose in the sequel that $r_{n}=1$. The continuity of the real valued functions $F$ at least on the set

$$
\Omega=\left[t_{0}, \infty\right) \times\left\{y=\left(y_{1}, y_{2}, \ldots, y_{N}\right) \in \mathbf{R}^{N}:(\forall i) y_{i}>0 \text { or }(\forall i) y_{i}<0\right\}
$$

and $g_{i}(i=1,2, \ldots, N)$ and $b$ on $\left[t_{0}, \infty\right)$ as well as sufficient smoothness for the existence of solutions of $(E, \delta)$ on an infinite subinterval of $\left[t_{0}, \infty\right)$ will be assumed without mentioning it any further. In what follows the term "solution" is always used only for such solutions $x(t)$ of $(E, \delta)$ which are defined for all large $t$. The oscillatory character is considered in the usual sense, that is a continuous real valued function which is defined on an interval of the form $\left[T_{0}, \infty\right)$ is called oscillatory if it has no last zero, and otherwise it is called nonoscillatory.

For real valued functions defined on subsets of the space $\mathbf{R}^{N}$ monotonicity will be considered with respect to the order in $\mathbf{R}^{N}$ defined by the usual positive cone

$$
\left\{y=\left(y_{1}, y_{2}, \ldots, y_{N}\right) \in \mathbf{R}^{N}:(\forall i) y_{i} \geqslant 0\right\} .
$$

Throughout the paper the function $F$ is supposed to be increasing on the set $\Omega$ with respect to the second variable. Moreover, conditions (i) and (ii) below will be assumed in the sequel without mentioning them any further:

(i) For every $i=1,2, \ldots, N$

$$
\lim _{t \rightarrow \infty} g_{i}(t)=\infty
$$

(ii) For each $t \geqslant t_{0}$ and every $y=\left(y_{1}, y_{2}, \ldots, y_{N}\right) \in \mathbf{R}^{N}$

$$
(\forall i) y_{i}>0 \Rightarrow F(t ; y) \geqslant 0
$$

and

$$
(\forall i) y_{i}<0 \Rightarrow F(t ; y) \leqslant 0 \text {. }
$$


Finally, for the sake of brevity, for any integer $m$ with $0 \leqslant m \leqslant n-1$ we introduce the function $R_{m}$ defined by

$$
R_{m}(t)=\left\{\begin{array}{l}
1, \quad \text { if } m=0 \\
\int_{t_{0}}^{t=s_{0}} \frac{1}{r_{1}\left(s_{1}\right)} \int_{t_{0}}^{s_{1}} \frac{1}{r_{2}\left(s_{2}\right)} \cdots \int_{t_{0}}^{s_{m-1}} \frac{1}{r_{m}\left(s_{m}\right)} d s_{m} \cdots d s_{2} d s_{1}, \quad \text { if } m>0
\end{array}\right.
$$

for all $t \geqslant t_{0}$.

For such differential equations involving the operator $D_{r}^{(n)}$ there is recently an increasing interest in studying the oscillatory and asymptotic behavior. We choose to refer here the papers by Kusano and Onose (1976a, 1976b), Philos (1977, 1978a, 1978b), Philos and Staikos (1979, 1980), Staikos and Philos (1977a, 1977b, 1978) and Trench (1975). As one can be seen in the book of Coppel (1971), the class of operators $D_{r}^{(n)}$ property contains the disconjugate operators $L$,

$$
L x=x^{(n)}+p_{1} x^{(n-1)}+\cdots+p_{n} x \text { ( } p_{i} \text { continuous functions). }
$$

The fundamental purpose of this paper is to find for any integer $m, 0 \leqslant m \leqslant n$ -1 , a necessary and sufficient condition (depending on $m$ ) in order to have at least one (nonoscillatory) solution $x$ of the differential equation $(E, \delta)$ with $b=0$ so that

$$
\lim _{t \rightarrow \infty}\left(D_{r}^{(m)} x\right)(t)=\lim _{t \rightarrow \infty} \frac{\left(D_{r}^{(0)} x\right)(t)}{R_{m}(t)}=L, \quad L \in \mathbf{R}-\{0\} .
$$

Necessary and sufficient conditions are established usually in order that differential equations with deviating arguments or functional differential equations of some forms have at least one (bounded nonoscillatory) solution whose the limit at $\infty$ exists in $\mathbf{R}-\{0\}$ [see, for example, Kusano and Onose (1975), Philos (1978a) and Staikos and Sficas (1972)]. The sufficiency of these conditions is proved by the fixed point technique based on the Schauder theorem [see Schauder (1930)] or the Tychonoff theorem [see Tychonoff (1935)]. For $n$-th order retarded differential equations of some special forms necessary and sufficient conditions are also given in order to have at least one (nonoscillatory) solution asymptotic to $L t^{n-1}$ as $t \rightarrow \infty$ for some nonzero real number $L$ [see Coffman and Wong (1972), Ladas (1971), Marušiak (1973), Onose (1973) and Grammatikopoulos, Sficas and Staikos (1978)]. The sufficiency in these papers was proved under a rather restrictive assumption referred to the existence of a solution defined for all large $t$ and satisfying an initial value condition. For any integer $m, 0 \leqslant m \leqslant n-1$, Kusano and Onose (1975) give a necessary condition (depending on $m$ ) in order for a class of retarded differential equations to have at least one (nonoscillatory) solution asymptotic to $L t^{m}$ as $t \rightarrow \infty$ for some $L \in \mathbf{R}-\{0\}$. They also make the conjecture that this condition is also sufficient. In this paper we deal, in a general setting, with this conjecture and we prove that it is true. The results obtained 
include some recent ones by Philos (1978a) and, moreover, these are new even in the case where $r_{0}=r_{1}=\cdots=r_{n-1}=1$. More comments on the results of this paper are given in the last section.

\section{Main results}

First of all, we shall prove a lemma, which is motivated by the following elementary result: If $\lim _{t \rightarrow \infty} h^{(m)}(t)$ exists in the extended real line $\mathbf{R}^{*}=\mathbf{R} \cup$ $\{-\infty, \infty\}$, then so does $\lim _{t \rightarrow \infty} m ! h(t) / t^{m}$ and, moreover, these limits are equal.

LeMMA 2.1. Let $m, 0 \leqslant m \leqslant n-1$, be an integer such that:

$\left(C_{1}\right)$ If $m>0$, then for every $i=1,2, \ldots, m$

$$
\int^{\infty} \frac{d t}{r_{i}(t)}=\infty
$$

Moreover, let $h$ be a function whose the r-derivative $D_{r}^{(m)} h$ exists in an interval $[T, \infty) T \geqslant t_{0}$. Then we have:

$\alpha)$ If $\lim _{t \rightarrow \infty}\left(D_{r}^{(m)} h\right)(t)$ exists in $\mathbf{R}^{*}-\{0\}$, then

$$
\lim _{t \rightarrow \infty} \frac{\left(D_{r}^{(0)} h\right)(t)}{R_{m}(t)}=\lim _{t \rightarrow \infty}\left(D_{r}^{(m)} h\right)(t) .
$$

$\beta)$ If $m>0$ and $D_{r}^{(m)} h$ is continuous on $[T, \infty)$, then

$$
\lim _{t \rightarrow \infty}\left(D_{r}^{(m)} h\right)(t)=0 \Rightarrow \lim _{t \rightarrow \infty} \frac{\left(D_{r}^{(0)} h\right)(t)}{R_{m}(t)}=0 .
$$

Proof. We shall prove the conclusion $\beta$ ) of the lemma, since $\alpha$ ) has been proved by Philos (1978b) for $m>0$ while for $m=0$ it is obvious. To this end, for any integers $i$ and $j, 0 \leqslant i \leqslant j \leqslant m$, and every $v, u$ with $v \geqslant u \geqslant t_{0}$ we define

$$
R_{i j}(v ; u)=\left\{\begin{array}{lll}
1, \quad \text { if } i=j, \\
\int_{u}^{v=s_{i}} \frac{1}{r_{i+1}\left(s_{i+1}\right)} \int_{u}^{s_{i+1}} \frac{1}{r_{i+2}\left(s_{i+2}\right)} & \cdots & \int_{u}^{s_{j-1}} \frac{1}{r_{j}\left(s_{j}\right)} d s_{j} \cdots \\
& \cdots d s_{i+2} d s_{i+1}, \quad \text { if } i<j .
\end{array}\right.
$$

Now, we assume that $D_{r}^{(m)} h$ is continuous on $[T, \infty)$ and $\lim _{t \rightarrow \infty}\left(D_{r}^{(m)} h\right)(t)=0$. Let any $\varepsilon>0$ and let $T^{*}>T$ be chosen so that

$$
\left|\left(D_{r}^{(m)} h\right)(t)\right|<\varepsilon \text { for every } t \geqslant T^{*} .
$$


For any $t \geqslant T^{*}$, it is easy to derive the following generalization of the Taylor formula

$$
\begin{aligned}
\left(D_{r}^{(0)} h\right)(t) & =\sum_{j=0}^{m-1}\left(D_{r}^{(j)} h\right)\left(T^{*}\right) R_{0 j}\left(t ; T^{*}\right) \\
& +\int_{T^{*}}^{t=s_{0}} \frac{1}{r_{1}\left(s_{1}\right)} \int_{T^{*}}^{s_{1}} \frac{1}{r_{2}\left(s_{2}\right)} \cdots \int_{T^{*}}^{s_{m-1}} \frac{1}{r_{m}\left(s_{m}\right)}\left(D_{r}^{(m)} h\right)\left(s_{m}\right) d s_{m} \cdots d s_{2} d s_{1} .
\end{aligned}
$$

From this formula we obtain

$$
\frac{\left|\left(D_{r}^{(0)} h\right)(t)\right|}{R_{m}(t)} \leqslant \sum_{j=0}^{m-1}\left|\left(D_{r}^{(j)} h\right)\left(T^{*}\right)\right| \frac{R_{0 j}\left(t ; T^{*}\right)}{R_{m}(t)}+\varepsilon \frac{R_{0 m}\left(t ; T^{*}\right)}{R_{m}(t)}
$$

for every $t \geqslant T^{*}$. But, since from condition $\left(\mathrm{C}_{1}\right)$ it follows that

$$
\begin{array}{ll}
\lim _{t \rightarrow \infty} R_{i j}\left(t ; T^{*}\right)=\infty & (0 \leqslant i<j \leqslant m), \\
\lim _{t \rightarrow \infty} R_{i m}\left(t ; t_{0}\right)=\infty & (0 \leqslant i \leqslant m-1),
\end{array}
$$

we can apply the De L'Hospital rule to verify that

$$
\lim _{t \rightarrow \infty} \frac{R_{0 j}\left(t ; T^{*}\right)}{R_{m}(t)}=0 \quad(j=0,1, \ldots, m-1) \quad \text { and } \quad \lim _{t \rightarrow \infty} \frac{R_{0 m}\left(t ; T^{*}\right)}{R_{m}(t)}=1 .
$$

Thus, we have

$$
\limsup _{t \rightarrow \infty} \frac{\left|\left(D_{r}^{(0)} h\right)(t)\right|}{R_{m}(t)} \leqslant \varepsilon
$$

and consequently, since $\varepsilon$ is arbitrary,

$$
\lim _{t \rightarrow \infty} \frac{\left(D_{r}^{(0)} h\right)(t)}{R_{m}(t)}=0 .
$$

To obtain our first theorem (Theorem 2.1) we shall apply the fixed point technique based on the well-known Schauder theorem [see Schauder (1930)].

The SChAUder TheOrem. Let $E$ be $a$ Banach space and $X$ any nonempty convex and closed subset of $E$. If $S$ is a continuous mapping of $X$ into itself and $S X$ is relatively compact, then the mapping $S$ has at least one fixed point (that is there exists an $x \in X$ with $x=S x$ ).

A set $-\mathscr{F}$ of real valued functions defined on the interval $[T, \infty)$ is said to be equiconvergent at $\infty$ if all functions in $\mathscr{F}$ are convergent in $\mathbf{R}$ at the point $\infty$ and, moreover, for every $\varepsilon>0$ there exists a $T^{\prime} \geqslant T$ such that, for all functions $f$ in $\mathscr{F}$,

$$
t \geqslant T^{\prime} \Rightarrow\left|f(t)-\lim _{s \rightarrow \infty} f(s)\right|<\varepsilon .
$$


Let, now, $B([T, \infty))$ be the Banach space of all continuous and bounded real valued functions on the interval $[T, \infty)$, endowed with the usual sup-norm \|\| . We need the following compactness criterion for subsets of $B([T, \infty))$, which is a corollary of the Arzelà-Ascoli theorem. For a proof of this criterion we refer to Staikos (1976).

COMPACTNESS CRITERION. Let $\mathscr{F}$ be an equicontinuous and uniformly bounded subset of the Banach space $B([T, \infty))$. If $\mathscr{F}$ is equiconvergent at $\infty$, it is also relatively compact.

THEOREM 2.1. Let $m, 0 \leqslant m \leqslant n-1$, be an integer such that the condition $\left(\mathrm{C}_{1}\right)$ and the following are satisfied:

$\left(\mathrm{C}_{2}\right)$ There exists an n-times continuously $r$-differentiable function $w$ on $\left[t_{0}, \infty\right)$ with $D_{r}^{(n)} w=b$ and such that

$$
A=\limsup _{t \rightarrow \infty} \frac{\left|\left(D_{r}^{(0)} w\right)(t)\right|}{R_{m}(t)}<\infty .
$$

$\left(\mathrm{C}_{3}\right)$ For some constant $c$ with $|c|>2 A$,

$$
\left\{\begin{array}{l}
\int^{\infty}\left|F\left(t ; c \frac{R_{n-1}}{r_{0}}\langle g(t)\rangle\right)\right| d t<\infty, \quad \text { if } m=n-1 \\
\int^{\infty} \frac{1}{r_{m+1}\left(s_{m+1}\right)} \cdots \int_{s_{n-2}}^{\infty} \frac{1}{r_{n-1}\left(s_{n-1}\right)} \int_{s_{n-1}}^{\infty}\left|F\left(s ; c \frac{R_{m}}{r_{0}}\langle g(s)\rangle\right)\right| d s d s_{n-1} \cdots \\
\cdots d s_{m+1}<\infty, \quad \text { if } m<n-1 .
\end{array}\right.
$$

Then for every number $L$ with $L c>0$ and $|c| / 2<|L|<|c|-A$ there exists $a$ (nonoscillatory) solution $x$ of the differential equation $(\mathrm{E}, \delta)$ such that

$$
\lim _{t \rightarrow \infty}\left[\left(D_{r}^{(m)} x\right)(t)-\left(D_{r}^{(m)} w\right)(t)\right]=L
$$

and, if $m<n-1$,

$$
\lim _{t \rightarrow \infty}\left[\left(D_{r}^{(j)} x\right)(t)-\left(D_{r}^{(j)} w\right)(t)\right]=0 \quad(j=m+1, \ldots, n-1) .
$$

Proof. For $m=0$ the theorem specializes to a theorem due to Philos (1978a). So, we restrict ourselves in the case where $m>0$.

Let $L$ be a real number with $L c>0$ and $|c| / 2<|L|<|c|-A$. Without loss of generality, we suppose that $c$ is positive, since the substitution $z=-x$ transforms $(\mathrm{E}, \delta)$ into an equation of the same form satisfying the assumptions of the theorem with $-c$ in place of $c$. Moreover, we assume that

$$
\frac{c}{2}<L<c-B,
$$


where

$$
B=\sup _{t \geqslant T_{0}} \frac{\left|\left(D_{r}^{(0)} w\right)(t)\right|}{R_{m}(t)}
$$

for some $T_{0}>t_{0}$. By conditions (i) and $\left(\mathrm{C}_{3}\right)$, we choose a $T \geqslant T_{0}$ so that

$$
g_{i}(t) \geqslant T_{0} \text { for every } t \geqslant T(i=1,2, \ldots, N)
$$

and

(1)

$$
\left\{\begin{array}{l}
\int_{T}^{\infty} F\left(t ; c \frac{R_{n-1}}{r_{0}}\langle g(t)\rangle\right) d t \leqslant c-L-B, \quad \text { if } m=n-1, \\
\int_{T}^{\infty} \frac{1}{r_{m+1}\left(s_{m+1}\right)} \cdots \int_{s_{n-2}}^{\infty} \frac{1}{r_{n-1}\left(s_{n-1}\right)} \int_{s_{n-1}}^{\infty} F\left(s ; c \frac{R_{m}}{r_{0}}\langle g(s)\rangle\right) d s d s_{n-1} \cdots \\
\cdots d s_{m+1} \leqslant c-L-B, \text { if } m<n-1 .
\end{array}\right.
$$

Let, now, the Banach space $E=\left\{h: r_{0} h / R_{m} \in B([T, \infty))\right\}$, endowed with the norm $f \nmid$ defined by

$$
\nmid h \nmid=\left\|\frac{r_{0}}{R_{m}}\right\| \text {. }
$$

Moreover, let $X$ be the set of all functions $x \in E$ with

$$
\left|\frac{\left(D_{r}^{(0)} x\right)(t)}{R_{m}(t)}-L\right| \leqslant c-L \quad \text { for every } t \geqslant T \text {. }
$$

Obviously, $L R_{m} / r_{0} \in E$ and $X=\left\{x \in E:\left\{x-L R_{m} / r_{0} \mid \leqslant c-L\right\}\right.$ is a closed ball in the normed space $E$. Therefore, $X$ is a nonempty, convex and closed subset of $E$.

If $x \in X$, by the definition of the set $X$, we obviously have

$$
0<\hat{x}(t) \leqslant c \frac{R_{m}}{r_{0}}(t) \text { for every } t \geqslant T_{0},
$$

where

$$
\hat{x}(t)=\left\{\begin{array}{l}
x(t), \quad \text { if } t \geqslant T \\
\frac{\left(D_{r}^{(0)} x\right)(T)}{R_{m}(T)} \frac{R_{m}(t)}{r_{0}(t)}, \quad \text { if } T_{0} \leqslant t \geqslant T
\end{array}\right.
$$

and consequently, in view of (ii) and the increasing character of the function $F$, we obtain

$$
0 \leqslant F(t ; \hat{x}\langle g(t)\rangle) \leqslant F\left(t ; c \frac{R_{m}}{r_{0}}\langle g(t)\rangle\right)
$$


for all $t \geqslant T$. Thus, because of condition $\left(\mathrm{C}_{3}\right)$, for any $x \in X$ and every $t \geqslant T$

$$
\begin{aligned}
& \int_{t}^{\infty} F(s ; \hat{x}\langle g(s)\rangle) d s<\infty, \quad \text { if } m=n-1, \\
& \int_{t}^{\infty} \frac{1}{r_{m+1}\left(s_{m+1}\right)} \cdots \int_{s_{n-2}}^{\infty} \frac{1}{r_{n-1}\left(s_{n-1}\right)} \int_{s_{n-1}}^{\infty} F(s ; \hat{x}\langle g(s)\rangle) d s d s_{n-1} \cdots \\
& \cdots d s_{m+1}<\infty, \quad \text { if } m<n-1
\end{aligned}
$$

and so, it is easy to see that for $t \geqslant T$ the formula

$$
\begin{aligned}
& (S x)(t)=L \frac{R_{m}(t)}{r_{0}(t)}+w(t) \\
& +\frac{\delta(-1)^{n-1-m}}{r_{0}(t)} \cdot\left\{\begin{array}{r}
\int_{T}^{t=s_{0}} \frac{1}{r_{1}\left(s_{1}\right)} \int_{T}^{s_{1}} \frac{1}{r_{2}\left(s_{2}\right)} \cdots \int_{T}^{s_{n-2}} \frac{1}{r_{n-1}\left(s_{n-1}\right)} \int_{s_{n-1}}^{\infty} F(s ; \hat{x}\langle g(s)\rangle) \\
\times d s d s_{n-1} \cdots d s_{2} d s_{1}, \quad \text { if } m=n-1 \\
\int_{T}^{t=s_{0}} \frac{1}{r_{1}\left(s_{1}\right)} \int_{T}^{s_{1}} \frac{1}{r_{2}\left(s_{2}\right)} \cdots \int_{T}^{s_{m-1}} \frac{1}{r_{m}\left(s_{m}\right)} \int_{s_{m}}^{\infty} \frac{1}{r_{m+1}\left(s_{m+1}\right)} \cdots \\
\cdots \int_{s_{n-2}}^{\infty} \frac{1}{r_{n-1}\left(s_{n-1}\right)} \int_{s_{n-1}}^{\infty} F\left(s ; \hat{x}\langle g(s)\rangle d s d s_{n-1} \cdots\right. \\
\cdots d s_{m+1} d s_{m} \cdots d s_{2} d s_{1}, \quad \text { if } m<n-1
\end{array}\right.
\end{aligned}
$$

introduces a mapping $S: X \rightarrow E$. The mapping $S$ satisfies the assumptions of the Schauder theorem. Namely, it satisfies the following:

a) $S X \subseteq X$.

In fact, taking into account (2) and (1), for any $x \in X$ and every $t \geqslant T$ we obtain

$$
\begin{aligned}
& \left|\frac{\left(D_{r}^{(0)}(S x)\right)(t)}{R_{m}(t)}-L\right| \leqslant \frac{\left|\left(D_{r}^{(0)} w\right)(t)\right|}{R_{m}(t)} \\
& +\frac{1}{R_{m}(t)} \cdot\left\{\begin{array}{r}
\int_{T}^{t=s_{0}} \frac{1}{r_{1}\left(s_{1}\right)} \int_{T}^{s_{1}} \frac{1}{r_{2}\left(s_{2}\right)} \cdots \int_{T}^{s_{n-2}} \frac{1}{r_{n-1}\left(s_{n-1}\right)} \int_{s_{n-1}}^{\infty} F(s ; \hat{x}\langle g(s)\rangle) \\
\int_{T}^{t=s_{0}} \frac{1}{r_{1}\left(s_{1}\right)} \int_{T}^{s_{1}} \frac{1}{r_{2}\left(s_{2}\right)} \cdots \int_{T}^{s_{m-1}} \frac{1}{r_{m}\left(s_{m}\right)} \int_{s_{m}}^{\infty} \frac{1}{r_{m+1}\left(s_{m+1}\right)} \cdots \\
\cdots \int_{s_{n-2}}^{\infty} \frac{1}{r_{n-1}\left(s_{n-1}\right)} \int_{s_{n-1}}^{\infty} F(s ; \hat{x}\langle g(s)\rangle) d s d s_{n-1} \cdots d s_{m+1} d s_{m} \cdots \\
\cdots d s_{2} d s_{1}, \quad \text { if } m<n-1
\end{array}\right.
\end{aligned}
$$




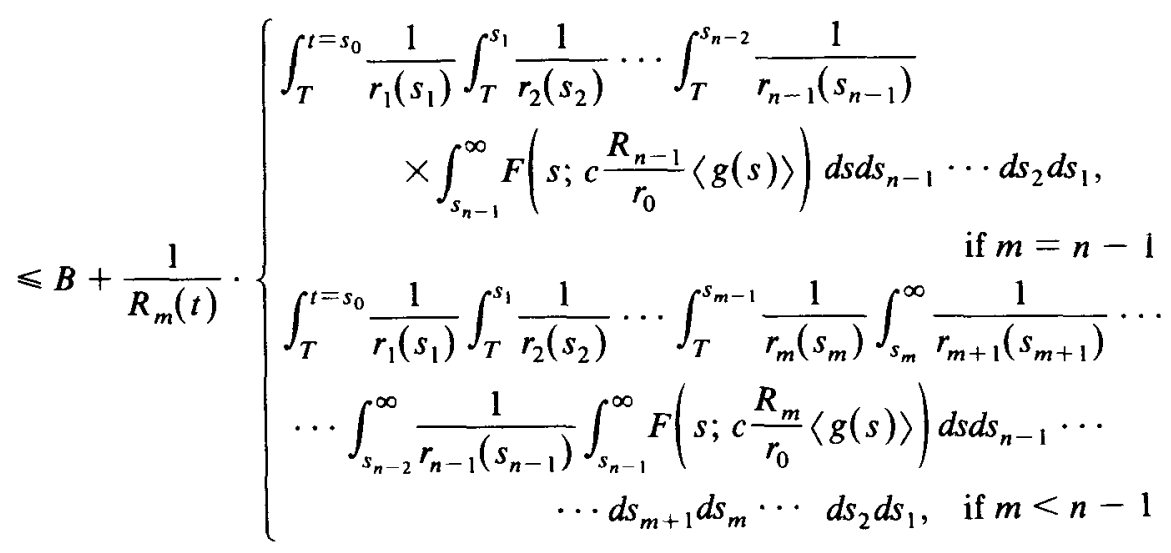$$
\leqslant B+(c-L-B)\left[\frac{1}{R_{m}(t)} \int_{T}^{t=s_{0}} \frac{1}{r_{1}\left(s_{1}\right)} \int_{T}^{s_{1}} \frac{1}{r_{2}\left(s_{2}\right)} \cdots \int_{T}^{s_{m-1}} \frac{1}{r_{m}\left(s_{m}\right)} d s_{m} \cdots\right.
$$$$
\left.\cdots d s_{2} d s_{1}\right]
$$

$\leqslant B+(c-L-B)=c-L$.

\section{B) $S X$ is relatively compact.}

Because of the fact that $S X \subseteq X$ and the definition of the set $X$, for any $x \in X$ and for $f=\left(D_{r}^{(0)}(S x)-D_{r}^{(0)} w\right) / R_{m}$ we have

$$
|f(t)| \leqslant \frac{\left|\left(D_{r}^{(0)}(S x)\right)(t)\right|}{R_{m}(t)}+\frac{\left|\left(D_{r}^{(0)} w\right)(t)\right|}{R_{m}(t)} \leqslant c+B, \quad t \geqslant T
$$

that is $\|f\| \leqslant c+B$. Therefore, the set

$$
\mathscr{F}=\left\{\frac{D_{r}^{(0)}(S x)}{R_{m}}-\frac{D_{r}^{(0)} w}{R_{m}}: x \in X\right\}
$$

is a uniformly bounded subset or the Banach space $B([T, \infty))$. Furthermore, if $x \in X$ and $f=\left(D_{r}^{(0)}(S x)-D_{r}^{(0)} w\right) / R_{m}$, then, taking into account (2), for every $t \geqslant T$ we obtain

$$
|f(t)-L|=\left|\left[\frac{\left(D_{r}^{(0)}(S x)\right)(t)}{R_{m}(t)}-\frac{\left(D_{r}^{(0)} w\right)(t)}{R_{m}(t)}\right]-L\right|
$$




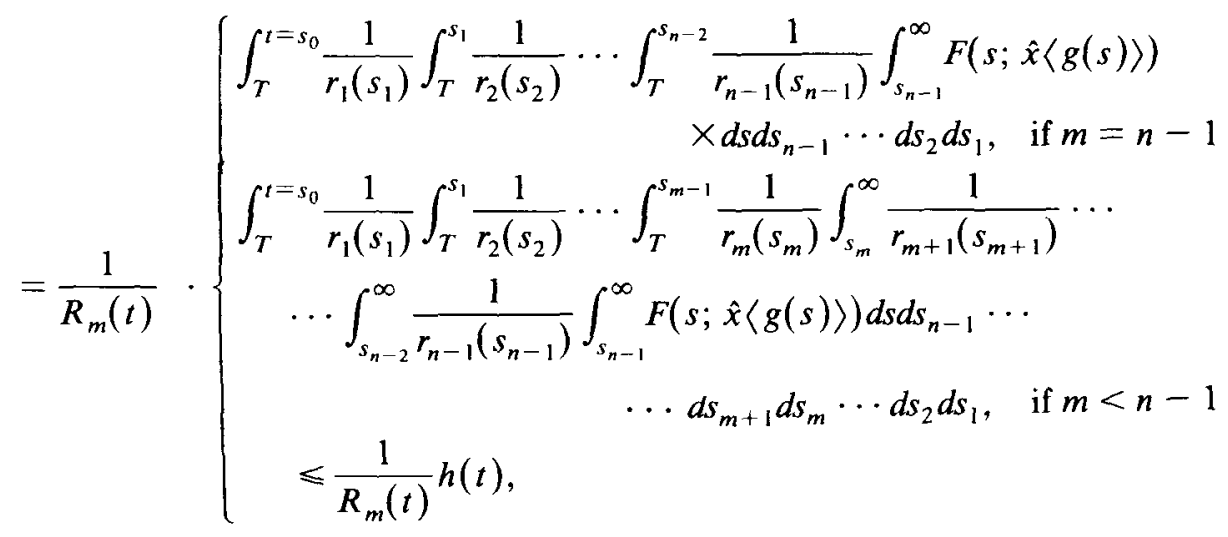

where

$$
\begin{gathered}
h(t)=\frac{1}{r_{0}(t)} \int_{T}^{t=s_{0}} \frac{1}{r_{1}\left(s_{1}\right)} \int_{T}^{s_{1}} \frac{1}{r_{2}\left(s_{2}\right)} \cdots \int_{T}^{s_{n-2}} \frac{1}{r_{n-1}\left(s_{n-1}\right)} \\
\quad \times \int_{s_{n-1}}^{\infty} F\left(s ; c \frac{R_{n-1}}{r_{0}}\langle g(s)\rangle\right) d s d s_{n-1} \cdots d s_{2} d s_{1}, \quad \text { if } m=n-1, \text { and } \\
h(t)=\frac{1}{r_{0}(t)} \int_{T}^{t=s_{0}} \frac{1}{r_{1}\left(s_{1}\right)} \int_{T}^{s_{1}} \frac{1}{r_{2}\left(s_{2}\right)} \cdots \int_{T}^{s_{m-1}} \frac{1}{r_{m}\left(s_{m}\right)} \int_{s_{m}}^{\infty} \frac{1}{r_{m+1}\left(s_{m+1}\right)} \cdots \\
\quad \cdots \int_{s_{n-2}}^{\infty} \frac{1}{r_{n-1}\left(s_{n-1}\right)} \int_{s_{n-1}}^{\infty} F\left(s ; c \frac{R_{m}}{r_{0}}\langle g(s)\rangle\right) d s d s_{n-1} \cdots d s_{m+1} d s_{m} \cdots d s_{2} d s_{1},
\end{gathered}
$$

if $m<n-1$. Namely, for any $f \in \mathscr{F}$ and every $t \geqslant T$

$$
|f(t)-L| \leqslant \frac{r_{0}(t)}{R_{m}(t)} h(t) .
$$

But,

$$
\lim _{t \rightarrow \infty} \frac{r_{0}(t)}{R_{m}(t)} h(t)=0 .
$$

Indeed, we have

$$
\left(D_{r}^{(m)} h\right)(t)=\left\{\begin{array}{r}
\int_{t}^{\infty} F\left(s ; c \frac{R_{n-1}}{r_{0}}\langle g(s)\rangle\right) d s, \quad \text { if } m=n-1 \\
\int_{t}^{\infty} \frac{1}{r_{m+1}\left(s_{m+1}\right)} \cdots \int_{s_{n-2}}^{\infty} \frac{1}{r_{n-1}\left(s_{n-1}\right)} \int_{s_{n-1}}^{\infty} F\left(s ; c \frac{R_{m}}{r_{0}}\langle g(s)\rangle\right) \\
\times d s d s_{n-1} \cdots d s_{m+1}, \quad \text { if } m<n-1
\end{array}\right.
$$


for all $t \geqslant T$. This gives

$$
\lim _{t \rightarrow \infty}\left(D_{r}^{(m)} h\right)(t)=0
$$

and so, by Lemma 2.1, we obtain (4). Because of (4), from (3) it follows that the set $\mathscr{F}$ is equiconvergent at $\infty$. Now, we shall prove that $\mathscr{F}$ is equicontinuous. For this purpose, it is enough to verify that for any $f \in \mathcal{F}$ and every $t_{1}, t_{2}$ with $T \leqslant t_{1} \leqslant t_{2}$

$$
\left|f\left(t_{2}\right)-f\left(t_{1}\right)\right| \leqslant \frac{2(c-L-B)}{R_{m}(t)}\left[R_{m}\left(t_{2}\right)-R_{m}\left(t_{1}\right)\right] .
$$

For $f=\left(D_{r}^{(0)}(S x)-D_{r}^{(0)} w\right) / R_{m}, x \in X$, by taking into account (2) and (1), the last inequality can be derived as follows

$$
\begin{aligned}
& \left|f\left(t_{2}\right)-f\left(t_{1}\right)\right| \leqslant\left|\frac{1}{R_{m}\left(t_{2}\right)}-\frac{1}{R_{m}\left(t_{1}\right)}\right| \\
& \iint_{T}^{t_{1}=s_{0}} \frac{1}{r_{1}\left(s_{1}\right)} \int_{T}^{s_{1}} \frac{1}{r_{2}\left(s_{2}\right)} \cdots \int_{T}^{s_{n-2}} \frac{1}{r_{n-1}\left(s_{n-1}\right)} \\
& \times \int_{s_{n-1}}^{\infty} F(s ; \hat{x}\langle g(s)\rangle) d s d s_{n-1} \cdots d s_{2} d s_{1}, \quad \text { if } m=n-1 \\
& \left\{\int_{T}^{t_{1}=s_{0}} \frac{1}{r_{1}\left(s_{1}\right)} \int_{T}^{s_{1}} \frac{1}{r_{2}\left(s_{2}\right)} \cdots \int_{T}^{s_{m-1}} \frac{1}{r_{m}\left(s_{m}\right)}\right. \\
& \times \int_{s_{m}}^{\infty} \frac{1}{r_{m+1}\left(s_{m+1}\right)} \cdots \int_{s_{n-2}}^{\infty} \frac{1}{r_{n-1}\left(s_{n-1}\right)} \int_{s_{n-1}}^{\infty} F(s ; \hat{x}\langle g(s)\rangle) \\
& \times d s d s_{n-1} \cdots d s_{2} d s_{1} \text {, if } m<n-1
\end{aligned}
$$

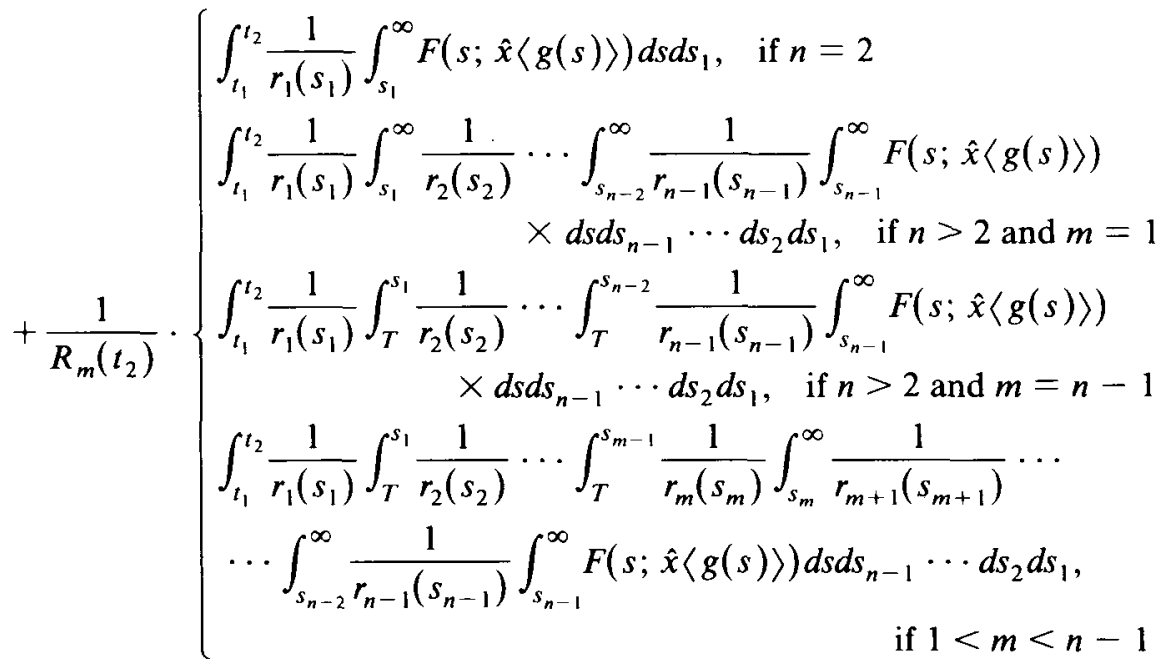


306

Ch. G. Philos, Y. G. Sficas and V. A. Staikos

[12]

$$
\begin{aligned}
& \leqslant \frac{R_{m}\left(t_{2}\right)-R_{m}\left(t_{1}\right)}{R_{m}(t)} \frac{1}{R_{m}\left(t_{1}\right)} \\
& \times\left\{\begin{aligned}
\int_{T}^{t_{1}=s_{0}} & \frac{1}{r_{1}\left(s_{1}\right)} \int_{T}^{s_{1}} \frac{1}{r_{2}\left(s_{2}\right)} \cdots \int_{T}^{s_{n-2}} \frac{1}{r_{n-1}\left(s_{n-1}\right)} \\
& \times \int_{s_{n-1}}^{\infty} F\left(s ; c \frac{R_{n-1}}{r_{0}}\langle g(s)\rangle\right) d s d s_{n-1} \cdots d s_{2} d s_{1}, \quad \text { if } m=n-1 \\
\int_{T}^{t_{1}=s_{0}} & \frac{1}{r_{1}\left(s_{1}\right)} \int_{T}^{s_{1}} \frac{1}{r_{2}\left(s_{2}\right)} \cdots \int_{T}^{s_{m-1}} \frac{1}{r_{m}\left(s_{m}\right)} \\
& \times \int_{s_{m}}^{\infty} \frac{1}{r_{m+1}\left(s_{m+1}\right)} \cdots \int_{s_{n-2}}^{\infty} \frac{1}{r_{n-1}\left(s_{n-1}\right)} \int_{s_{n-1}}^{\infty} F\left(s ; c \frac{R_{m}}{r_{0}}\langle g(s)\rangle\right)
\end{aligned}\right. \\
& +\frac{1}{R_{m}(T)} \cdot\left\{\begin{array}{l}
\int_{t_{1}}^{t_{2}} \frac{1}{r_{1}\left(s_{1}\right)} \int_{s_{1}}^{\infty} F\left(s ; c \frac{R_{1}}{r_{0}}\langle g(s)\rangle\right) d s d s_{1}, \quad \text { if } n=2 \\
\int_{t_{1}}^{t_{2}} \frac{1}{r_{1}\left(s_{1}\right)} \int_{s_{1}}^{\infty} \frac{1}{r_{2}\left(s_{2}\right)} \cdots \int_{s_{n-2}}^{\infty} \frac{1}{r_{n-1}\left(s_{n-1}\right)} \int_{s_{n-1}}^{\infty} F\left(s ; c \frac{R_{1}}{r_{0}}\langle g(s)\rangle\right) \\
\quad \times d s d s_{n-1} \cdots d s_{2} d s_{1}, \quad \text { if } n>2 \text { and } m=1 \\
\int_{t_{1}}^{t_{2}} \frac{1}{r_{1}\left(s_{1}\right)} \int_{T}^{s_{1}} \frac{1}{r_{2}\left(s_{2}\right)} \cdots \int_{T-2}^{s_{n-2}} \frac{1}{r_{n-1}\left(s_{n-1}\right)} \int_{s_{n-1}}^{\infty} F\left(s ; c \frac{R_{n-1}}{r_{0}}\langle g(s)\rangle\right) \\
\int_{t_{1}}^{t_{2}} \frac{1}{r_{1}\left(s_{1}\right)} \int_{T}^{s_{1}} \frac{1}{r_{2}\left(s_{2}\right)} \cdots \int_{T}^{s_{m-1}} \frac{1}{r_{m}\left(s_{m}\right)} \int_{s_{m}}^{\infty} \frac{1}{r_{m+1}\left(s_{m+1}\right)} \cdots \\
\cdots \int_{s_{n-2}}^{\infty} \frac{1}{r_{n-1}\left(s_{n-1}\right)} \int_{s_{n-1}}^{\infty} F\left(s ; c \frac{R_{m}}{r_{0}}\langle g(s)\rangle\right) d s d s_{n-1} \cdots d s_{2} d s_{1}, \\
\text { if } 1<m<n-1 .
\end{array}\right. \\
& \leqslant \frac{c-L-B}{R_{m}(T)}\left[\frac{1}{R_{m}\left(t_{1}\right)} \int_{T}^{t_{1}=s_{0}} \frac{1}{r_{1}\left(s_{1}\right)} \int_{T}^{s_{1}} \frac{1}{r_{2}\left(s_{2}\right)} \cdots\right. \\
& \left.\cdots \int_{T}^{s_{m-1}} \frac{1}{r_{m}\left(s_{m}\right)} d s_{m} \cdots d s_{2} d s_{1}\right]\left[R_{m}\left(t_{2}\right)-R_{m}\left(t_{1}\right)\right] \\
& +\frac{c-L-B}{R_{m}(T)}\left\{\begin{array}{l}
\int_{t_{1}}^{t_{2}} \frac{1}{r_{1}\left(s_{1}\right)} d s_{1}, \quad \text { if } m=1 \\
\int_{t_{1}}^{t_{2}} \frac{1}{r_{1}\left(s_{1}\right)} \int_{T}^{s_{1}} \frac{1}{r_{2}\left(s_{2}\right)} \cdots \int_{T}^{s_{m-1}} \frac{1}{r_{m}\left(s_{m}\right)} d s_{m} \cdots d s_{2} d s_{1}, \quad \text { if } m>1
\end{array}\right.
\end{aligned}
$$

https://doi.org/10.1017/S144678870002485X Published online by Cambridge University Press 


$$
\begin{aligned}
& \leqslant \frac{c-L-B}{R_{m}(T)}\left[R_{m}\left(t_{2}\right)-R_{m}\left(t_{1}\right)\right]+\frac{c-L-B}{R_{m}(T)}\left[R_{m}\left(t_{2}\right)-R_{m}\left(t_{1}\right)\right] \\
& =\frac{2 c-L-B}{R_{m}(T)}\left[R_{m}\left(t_{2}\right)-R_{m}\left(t_{1}\right)\right] .
\end{aligned}
$$

It has been proved that $\mathscr{F}$ is a uniformly bounded, equiconvergent at $\infty$ and equicontinuous subset of the Banach space $B([T, \infty))$. So, by the given compactness criterion, $\mathscr{F}$ is relatively compact. This, because of the definition of the norm $1\}$ in $E$ and the boundedness of the function $D_{r}^{(0)} w / R_{m}$, implies the relative compactness of $S X$.

$\gamma)$ The mapping $S$ is continuous.

Let $x \in X$ and $\left(x_{\nu}\right)$ be an arbitrary sequence in $X$ with $\lim x_{\nu}=x$ with respect to the norm $\nmid \nmid$ in $E$. By (2), for all $\nu$ and every $t \geqslant T$ we have

$$
F\left(t ; \hat{x}_{\nu}\langle g(t)\rangle\right) \leqslant F\left(t ; c \frac{R_{m}}{r_{0}}\langle g(t)\rangle\right)
$$

and consequently, because of condition $\left(\mathrm{C}_{3}\right)$, we can apply the Lebesgue dominated convergence theorem to obtain for $t \geqslant T$

$$
\lim _{\nu} \int_{t}^{\infty} F\left(s ; \hat{x}_{\nu}\langle g(s)\rangle\right) d s=\int_{t}^{\infty} F(s ; \hat{x}\langle g(s)\rangle) d s,
$$

if $m=n-1$, and

$$
\begin{aligned}
\lim _{\nu} \int_{t}^{\infty} & \frac{1}{r_{m+1}\left(s_{m+1}\right)} \cdots \int_{s_{n-2}}^{\infty} \frac{1}{r_{n-1}\left(s_{n-1}\right)} \int_{s_{n-1}}^{\infty} F\left(s ; \hat{x}_{\nu}\langle g(s)\rangle\right) d s d s_{n-1} \cdots d s_{m+1} \\
& =\int_{t}^{\infty} \frac{1}{r_{m+1}\left(s_{m+1}\right)} \cdots \int_{s_{n-2}}^{\infty} \frac{1}{r_{n-1}\left(s_{n-1}\right)} \int_{s_{n-1}}^{\infty} F(s ; \hat{x}\langle g(s)\rangle) d s d s_{n-1} \cdots d s_{m+1},
\end{aligned}
$$

if $m<n-1$. So, we have the pointwise convergence

$$
\lim _{v}\left(S x_{\nu}\right)(t)=(S x)(t), \quad t \geqslant T .
$$

Now, in order to prove that

$$
11-\lim S x_{v}=S x
$$

we consider any subsequence $\left(z_{\mu}\right)$ of $\left(S x_{\nu}\right)$. Then, because of the relative compactness of the set $S X$, there exist a subsequence $\left(\nu_{\lambda}\right)$ of $\left(z_{\mu}\right)$ and a $y \in E$ so that

$$
11-\lim \nu_{\lambda}=y \text {. }
$$

Since $\nmid\{$-convergence implies the pointwise one to the same limit function, we must always have $y=S x$. Thus, (5) and hence the continuity of the mapping $S$ is proved. 
Finally, by the Schauder theorem, there exists an $x \in X$ with $x=S x$. Then, in view of condition $\left(\mathrm{C}_{2}\right)$,

$$
\left(D_{r}^{(n)} x\right)(t)=\left(D_{r}^{(n)} w\right)(t)-\delta F(t ; \hat{x}\langle g(t)\rangle)=b(t)-\delta F(t ; \hat{x}\langle g(t)\rangle)
$$

for every $t \geqslant T$. Therefore, the fixed point $x$ of the mapping $S$ is a solution on $[T, \infty)$ of the equation $(\mathrm{E}, \delta)$. Moreover, this solution is the required one, since

$$
\begin{aligned}
& \mathbb{I}\left[\left(D_{r}^{(m)} x\right)(t)-\left(D_{r}^{(m)} w\right)(t)\right]-L \mid \\
& \quad=\left\{\begin{array}{l}
\int_{t}^{\infty} F(s ; \hat{x}\langle g(s)\rangle) d s, \quad \text { if } m=n-1 \\
\int_{t}^{\infty} \frac{1}{r_{m+1}\left(s_{m+1}\right)} \cdots \int_{s_{n-2}}^{\infty} \frac{1}{r_{n-1}\left(s_{n-1}\right)} \int_{s_{n-1}}^{\infty} F(s ; \hat{x}\langle g(s)\rangle) d s d s_{n-1} \cdots d s_{m+1},
\end{array}\right.
\end{aligned}
$$

$\rightarrow 0$ as $t \rightarrow \infty$

and, if $m<n-1$, for $j=m+1, \ldots, n-1$

$$
\begin{aligned}
& \left|\left(D_{r}^{(j)} x\right)(t)-\left(D_{r}^{(j)} w\right)(t)\right| \\
& \quad= \begin{cases}\int_{t}^{\infty} F(s ; \hat{x}\langle g(s)\rangle) d s, \quad \text { if } j=n-1 \\
\int_{t}^{\infty} \frac{1}{r_{j+1}\left(s_{j+1}\right)} \cdots \int_{s_{n-2}}^{\infty} \frac{1}{r_{n-1}\left(s_{n-1}\right)} \int_{s_{n-1}}^{\infty} F(s ; \hat{x}\langle g(s)\rangle) d s d s_{n-1} \cdots d s_{j+1},\end{cases}
\end{aligned}
$$

$\rightarrow 0$ as $t \rightarrow \infty$.

Corollary 2.1. Let $m, 0 \leqslant m \leqslant n-1$, be an integer such that the condition $\left(\mathrm{C}_{1}\right)$ and the following are satisfied:

$\left(\mathrm{C}_{2}^{\prime}\right)$ There exists an n-times continuously $r$-differentiable function $w$ on $\left[t_{0}, \infty\right)$ with $D_{r}^{(n)} w=b$ and $\lim _{t \rightarrow \infty}\left(D_{r}^{(m)} w\right)(t)=0$.

$\left(\mathrm{C}_{3}^{\prime}\right)$ For some nonzero constant $c$,

$$
\left\{\begin{array}{l}
\int^{\infty}\left|F\left(t ; c \frac{R_{n-1}}{r_{0}}\langle g(t)\rangle\right)\right| d t<\infty, \quad \text { if } m=n-1 \\
\int^{\infty} \frac{1}{r_{m+1}\left(s_{m+1}\right)} \cdots \int_{s_{n-2}}^{\infty} \frac{1}{r_{n-1}\left(s_{n-1}\right)} \int_{s_{n-1}}^{\infty}\left|F\left(s ; c \frac{R_{m}}{r_{0}}\langle g(s)\rangle\right)\right| d s d s_{n-1} \cdots \\
\cdots d s_{m+1}<\infty, \quad \text { if } m<n-1 .
\end{array}\right.
$$

Then for every real number $L$ with $L c>0$ and $|c| / 2<|L|<|c|$ there exists a (nonoscillatory) solution $x$ of the differential equation $(\mathrm{E}, \delta)$ with

$$
\lim _{t \rightarrow \infty}\left(D_{r}^{(m)} x\right)(t)=L .
$$


Proof. Since $\lim _{t \rightarrow \infty}\left(D_{r}^{(m)} w\right)(t)=0$, by Lemma 2.1, we have

$$
A=\limsup _{t \rightarrow \infty} \frac{\left|\left(D_{r}^{(0)} w\right)(t)\right|}{R_{m}(t)}=0 .
$$

So, the corollary follows immediately from Theorem 2.1

In particular, for $b=0$ and $w=0$, from Theorem 2.1 we can immediately obtain the following result concerning the differential equation $\left(\mathrm{E}_{0}, \boldsymbol{\delta}\right)$

$$
\left(D_{r}^{(n)} x\right)(t)+\delta F(t ; x\langle g(t)\rangle)=0 .
$$

Corollary 2.2. Let $m, 0 \leqslant m \leqslant n-1$, be an integer such that the conditions $\left(\mathrm{C}_{1}\right)$ and $\left(\mathrm{C}_{3}^{\prime}\right)$ are satisfied. Then for every real number $L$ with $L c>0$ and $|c| / 2<|L|<|c|$ there exists a (nonoscillatory) solution $x$ of the differential equation $\left(\mathrm{E}_{0}, \delta\right)$ such that

$$
\lim _{t \rightarrow \infty}\left(D_{r}^{(m)} x\right)(t)=L
$$

and, if $m<n-1$,

$$
\lim _{t \rightarrow \infty}\left(D_{r}^{(j)} x\right)(t)=0, \quad(j=m+1, \ldots, n-1) .
$$

In order to obtain the next theorem (Theorem 2.2) we make use of the iollowing elementary lemma given by Philos (1978a), which is motivated by the very simple result: If $\lim _{t \rightarrow \infty} u(t)$ is finite and $\lim _{t \rightarrow \infty} u^{\prime}(t)$ exists in $\mathbf{R}^{*}$, then $\lim _{t \rightarrow \infty} u^{\prime}(t)=0$.

LeMMA 2.2. Let $h$ be an n-times $r$-differentiable function on an interval $[T, \infty)$, $T \geqslant t_{0}$, such that $D_{r}^{(n)} h$ is of constant sign on $[T, \infty)$. Moreover, let $\lambda, 0 \leqslant \lambda \leqslant n-$ 2 , be an integer so that

$$
\int^{\infty} \frac{d t}{r_{\lambda+1}(t)}=\infty
$$

If $\lim _{t \rightarrow \infty}\left(D_{r}^{(\lambda)} h\right)(t)$ is finite, then

$$
\lim _{t \rightarrow \infty}\left(D_{r}^{(\lambda+1)} h\right)(t)=0 .
$$

THEOREM 2.2. Suppose that:

$\left(\mathrm{C}_{4}\right)$ For every $i=1,2, \ldots, n-1$

$$
\int^{\infty} \frac{d t}{r_{i}(t)}=\infty
$$


Let $m, 0 \leqslant m \leqslant n-1$, be an integer such that the following condition is satisfied:

$\left(\mathrm{C}_{5}\right)$ For every nonzero constant $c$ there exists an integer $k$ with $m \leqslant k \leqslant n-1$ and such that

$$
\left\{\begin{array}{l}
\int^{\infty}\left|F\left(t ; c \frac{R_{m}}{r_{0}}\langle g(t)\rangle\right)\right| d t=\infty, \quad \text { if } k=n-1 \\
\int^{\infty} \frac{1}{r_{k+1}\left(s_{k+1}\right)} \cdots \int_{s_{n-2}}^{\infty} \frac{1}{r_{n-1}\left(s_{n-1}\right)} \int_{s_{n-1}}^{\infty}\left|F\left(s ; c \frac{R_{m}}{r_{0}}\langle g(s)\rangle\right)\right| d s d s_{n-1} \cdots \\
\cdots d s_{k+1}=\infty, \quad \text { if } k<n-1 .
\end{array}\right.
$$

Then for every nonoscillatory solution $x$ of the differential equation $\left(\mathrm{E}_{0}, \delta\right)$ with $x(t)=O\left(R_{m}(t) / r_{0}(t)\right)$ as $t \rightarrow \infty$,

$$
\delta(-1)^{n+m} x(t)\left(D_{r}^{(m)} x\right)(t) \leqslant 0 \text { for all large } t
$$

and

$$
\lim _{t \rightarrow \infty}\left(D_{r}^{(j)} x\right)(t)=0 \text { monotonically } \quad(j=m, m+1, \ldots, n-1) .
$$

Proof. Let $x$ be a nonoscillatory solution on an interval $\left[T_{0}, \infty\right), T_{0}>t_{0}$, of the equation $\left(\mathrm{E}_{0}, \delta\right)$ with $x(t)=O\left(R_{m}(t) / r_{0}(t)\right)$ as $t \rightarrow \infty$. Without loss of generality, we suppose that $x(t) \neq 0$ for every $t \geqslant T_{0}$. Furthermore, we can assume that $x$ is positive, since the substitution $z=-x$ transforms $\left(\mathrm{E}_{0}, \delta\right)$ into an equation of the same form satisfying the assumptions of the theorem.

Next, by (i), we choose a $T \geqslant T_{0}$ so that

$$
g_{i}(t) \geqslant T_{0} \quad \text { for every } t \geqslant T \quad(i=1,2, \ldots, N) .
$$

Then, in view of (ii), equation $\left(\mathrm{E}_{0}, \delta\right)$ yields

$$
-\delta\left(D_{r}^{(n)} x\right)(t)=F(t ; x\langle g(t)\rangle) \geqslant 0
$$

for all $t \geqslant T$. Namely,

$$
\delta\left(D_{r}^{(n)} x\right)(t) \leqslant 0 \text { for every } t \geqslant T .
$$

Therefore, it follows that the functions $D_{r}^{(j)} x(j=m, m+1, \ldots, n-1)$ are eventually of constant sign. Without loss of generality, we can suppose that this holds true on the whole interval $[T, \infty)$.

By Lemma 2.1,

$$
\lim _{t \rightarrow \infty} \frac{\left(D_{r}^{(0)} x\right)(t)}{R_{m}(t)}=\lim _{t \rightarrow \infty}\left(D_{r}^{(m)} x\right)(t) .
$$

So, since $x$ is positive and $x(t)=O\left(R_{m}(t) / r_{0}(t)\right)$ as $t \rightarrow \infty$, we have

$$
\lim _{t \rightarrow \infty}\left|\left(D_{r}^{(m)} x\right)(t)\right|<\infty
$$


and consequently, in the case where $m<n-1$, we can consecutively apply Lemma 2.2 for $\lambda=m, m+1, \ldots, n-2$ to obtain

$$
\lim _{t \rightarrow \infty}\left(D_{r}^{(j)} x\right)(t)=0 \quad(j=m+1, \ldots, n-1) .
$$

We have

$$
\int_{T}^{\infty} \frac{1}{r_{m+1}(t)}\left|\left(D_{r}^{(m+1)} x\right)(t)\right| d t<\infty
$$

This follows immediately from (7) and

$$
\left|\left(D_{r}^{(m)} x\right)(t)-\left(D_{r}^{(m)} x\right)(T)\right|=\int_{T}^{t} \frac{1}{r_{m+1}(s)}\left|\left(D_{r}^{(m+1)} x\right)(s)\right| d s, \quad t \geqslant T .
$$

Moreover, if $m<n-1$, then for any $j=m+1, \ldots, n-1$ and every $t \geqslant T$

$$
\left|\left(D_{r}^{(j)} x\right)(t)\right| \geqslant \int_{t}^{\infty} \frac{1}{r_{j+1}(s)}\left|\left(D_{r}^{(j+1)} x\right)(s)\right| d s .
$$

Indeed, for all $u \geqslant t$

$$
\left|\left(D_{r}^{(j)} x\right)(t)\right|+\left|\left(D_{r}^{(j)} x\right)(u)\right| \geqslant \int_{t}^{u} \frac{1}{r_{j+1}(s)}\left|\left(D_{r}^{(j+1)} x\right)(s)\right| d s
$$

from which, by (8), we obtain (10). By using (9) and (10), it is easy to verify that

$$
\left\{\begin{array}{l}
\int_{T}^{\infty}\left|\left(D_{r}^{(n)} x\right)(t)\right| d t<\infty, \quad \text { if } m=n-1 \\
\int_{T}^{\infty} \frac{1}{r_{m+1}\left(s_{m+1}\right)} \cdots \int_{s_{n-2}}^{\infty} \frac{1}{r_{n-1}\left(s_{n-1}\right)} \int_{s_{n-1}}^{\infty}\left|\left(D_{r}^{(n)} x\right)(s)\right| d s d s_{n-1} \cdots \\
\cdots d s_{m+1}<\infty, \quad \text { if } m<n-1 .
\end{array}\right.
$$

Now, we shall prove that

$$
\lim _{t \rightarrow \infty}\left(D_{r}^{(m)} x\right)(t)=0
$$

To this end, we suppose the contrary, that is $\lim _{t \rightarrow \infty}\left(D_{r}^{(m)} x\right)(t)>0$. Then, by Lemma 2.1,

$$
\lim _{t \rightarrow \infty} \frac{\left(D_{r}^{(0)} x\right)(t)}{R_{m}(t)}>0
$$

and consequently there exists a positive constant $c$ such that

$$
x(t) \geqslant c \frac{R_{m}}{r_{0}}(t) \text { for every } t \geqslant T_{0} .
$$


Thus, in view of (ii) and the increasing character of $F$, from equation $\left(\mathrm{E}_{0}, \delta\right)$ we obtain

$$
-\delta\left(D_{r}^{(n)} x\right)(t)=F(t ; x\langle g(t)\rangle) \geqslant F\left(t ; c \frac{R_{m}}{r_{0}}\langle g(t)\rangle\right) \geqslant 0
$$

for all $t \geqslant T$. So, because of (11), for the considered (positive) constant $c$ we have

$$
\left\{\begin{array}{l}
\int_{T}^{\infty} F\left(t ; c \frac{R_{n-1}}{r_{0}}\langle g(t)\rangle\right) d t<\infty, \quad \text { if } m=n-1 \\
\int_{T}^{\infty} \frac{1}{r_{m+1}\left(s_{m+1}\right)} \cdots \int_{s_{n-2}}^{\infty} \frac{1}{r_{n-1}\left(s_{n-1}\right)} \int_{s_{n-1}}^{\infty} F\left(s ; c \frac{R_{m}}{r_{0}}\langle g(s)\rangle\right) d s d s_{n-1} \cdots \\
\cdots d s_{m+1}<\infty, \quad \text { if } m<n-1,
\end{array}\right.
$$

which contradicts $\left(\mathrm{C}_{5}\right)$.

Finally, because of (12) and (8), we must always have

$$
\left(D_{r}^{(j)} x\right)(t)\left(D_{r}^{(j+1)} x\right)(t) \leqslant 0 \quad \text { for every } t \geqslant T \quad(j=m, m+1, \ldots, n-1),
$$

which, by virtue of (6), easily gives

$$
\delta(-1)^{n+m}\left(D_{r}^{(m)} x\right)(t) \leqslant 0 \text { for every } t \geqslant T .
$$

Now, we state the following result by Philos (1978a) needed below in obtaining Corollary 2.3, which constitutes a basic "if and only if" criterion.

THEOREM 2.3. Let the condition $\left(\mathrm{C}_{4}\right)$ and the following be satisfied:

$\left(\mathrm{C}_{6}\right)$ For every nonzero constant $c$ there exists an integer $k, 0 \leqslant k \leqslant n-1$, such that

$$
\left\{\begin{array}{r}
\int^{\infty}\left|F\left(t ; c \frac{1}{r_{0}}\langle g(t)\rangle\right)\right| d t=\infty, \quad \text { if } k=n-1 \\
\int^{\infty} \frac{1}{r_{k+1}\left(s_{k+1}\right)} \cdots \int_{s_{n-2}}^{\infty} \frac{1}{r_{n-1}\left(s_{n-1}\right)} \int_{s_{n-1}}^{\infty}\left|F\left(s ; c \frac{1}{r_{0}}\langle g(s)\rangle\right)\right| d s d s_{n-1} \cdots \\
\cdots d s_{k+1}=\infty, \quad \text { if } k<n-1 .
\end{array}\right.
$$

Then every solution $x$ of the differential equation $\left(\mathrm{E}_{0},+1\right)$ [respectively, of the equation $\left.\left(\mathrm{E}_{0},-1\right)\right]$ with $x(t)=O\left(1 / r_{0}(t)\right)$ as $t \rightarrow \infty$ for $n$ even [resp. odd] is oscillatory, while for $n$ odd [resp. even] is either oscillatory or such that

$$
\lim _{t \rightarrow \infty}\left(D_{r}^{(j)} x\right)(t)=0 \text { monotonically }(j=0,1, \ldots, n-1) .
$$


Corollary 2.3. Let the condition $\left(\mathrm{C}_{4}\right)$ be satisfied and let $m, 0 \leqslant m \leqslant n-1$, be an integer. Then the condition $\left(\mathrm{C}_{3}^{\prime}\right)$ is a necessary and sufficient condition in order that the differential equation $\left(\mathrm{E}_{0}, \delta\right)$ have at least one (nonoscillatory) solution $x$ so that the $\lim _{t \rightarrow \infty}\left(D_{r}^{(m)} x\right)(t)$ exists in $\mathbf{R}-\{0\}$.

Proof. The sufficiency of the condition $\left(\mathrm{C}_{3}^{\prime}\right)$ is contained in Corollary 2.2. On the other hand, if $x$ is any (nonoscillatory) solution of the equation $\left(\mathrm{E}_{0}, \delta\right)$ such that the $\lim _{t \rightarrow \infty}\left(D_{r}^{(m)} x\right)(t)$ exists in $\mathbf{R}-\{0\}$, then, by Lemma 2.1 , we obviously have $x(t)=O\left(R_{m}(t) / r_{0}(t)\right)$ as $t \rightarrow \infty$. So, the necessity of the condition $\left(\mathrm{C}_{3}^{\prime}\right)$ can be obtained from Theorems 2.2 and 2.3.

\section{Applications}

Let the differential equation

$$
\left[r(t) x^{(n-l)}(t)\right]^{(l)}+\delta F(t ; x\langle g(t)\rangle)=b(t),
$$

where $l$ is an integer with $1 \leqslant l \leqslant n-1$ and $r$ a positive continuous function on the interval $\left[t_{0}, \infty\right)$. Obviously, this equation is obtained from equation $(\mathrm{E}, \delta)$ by setting

$$
r_{i}=1(i=0,1, \ldots, n-1 ; i \neq n-l) \text { and } r_{n-l}=r \text {. }
$$

With some manipulations we can formulate the results of Section 2 for the differential equation $(\hat{\mathrm{E}}, \delta)$. We omit doing it here and we restrict ourselves in the usual case where

$$
r_{0}=r_{1}=\cdots=r_{n-1}=1
$$

in order to better clarify the relevance of our main results. We note that in this case for any integer $m, 0 \leqslant m \leqslant n-1$, the condition $\left(C_{1}\right)$ holds by itself and we have

$$
R_{m}(t)=\left(t-t_{0}\right)^{m} / m !, \quad t \geqslant t_{0} .
$$

Moreover, the condition $\left(\mathrm{C}_{4}\right)$ holds too by itself. Corollaries 3.1, 3.2, 3.3, 3.4 and 3.5 below concern the differential equation

$$
x^{(n)}(t)+\delta F(t ; x\langle g(t)\rangle)=b(t)
$$

and follow from Theorem 2.1, Corollary 2.1, Corollary 2.2, Theorem 2.2 and Corollary 2.3 respectively. All these corollaries are new. 
COROLlaRY 3.1. Let $m, 0 \leqslant m \leqslant n-1$, be an integer such that the following conditions are satisfied:

$\left(\tilde{\mathrm{C}}_{2}\right)$ There exists an $n$-times continuously differentiable function $w$ on $\left[t_{0}, \infty\right)$ with $w^{(n)}=b$ and such that

$$
\tilde{A}=\limsup _{t \rightarrow \infty} \frac{|w(t)|}{t^{m}}<\infty .
$$

$\left(\tilde{\mathrm{C}}_{3}\right)$ For some constant $c$ with $|c|>2 \tilde{A}$,

$$
\int^{\infty} t^{n-1-m}\left|F\left(t ; c\left[g_{1}(t)\right]^{m}, c\left[g_{2}(t)\right]^{m}, \ldots, c\left[g_{N}(t)\right]^{m}\right)\right| d t<\infty .
$$

Then for every real number $L$ with $L c>0$ and $|c| / 2<|L|<|c|-\tilde{A}$ there exists $a$ (nonoscillatory) solution $x$ of the differential equation $(\tilde{\mathrm{E}}, \delta)$ such that

$$
\lim _{t \rightarrow \infty}\left[x^{(m)}(t)-w^{(m)}(t)\right]=L / m !
$$

and, if $m<n-1$,

$$
\lim _{t \rightarrow \infty}\left[x^{(j)}(t)-w^{(j)}(t)\right]=0 \quad(j=m+1, \ldots, n-1) .
$$

COROllary 3.2. Let $m, 0 \leqslant m \leqslant n-1$, be an integer such that the following conditions are satisfied:

$\left(\tilde{\mathrm{C}}_{2}^{\prime}\right)$ There exists an n-times continuously differentiable function $w$ on $\left[t_{0}, \infty\right)$ with $w^{(n)}=b$ and $\lim _{t \rightarrow \infty} w^{(m)}(t)=0$.

$\left(\tilde{\mathrm{C}}_{3}^{\prime}\right)$ For some nonzero constant $c$,

$$
\int^{\infty} t^{n-1-m}\left|F\left(t ; c\left[g_{1}(t)\right]^{m}, c\left[g_{2}(t)\right]^{m}, \ldots, c\left[g_{N}(t)\right]^{m}\right)\right| d t<\infty .
$$

Then for every real number $L$ with $L c>0$ and $|c| / 2<|L|<|c|$ there exists a (nonoscillatory) solution $x$ of the differential equation $(\tilde{\mathrm{E}}, \delta)$ with

$$
\lim _{t \rightarrow \infty} x^{(m)}(t)=L / m !
$$

Corollary. 3.3. Let $m, 0 \leqslant m \leqslant n-1$, be an integer such that the condition $\left(\tilde{\mathrm{C}}_{3}^{\prime}\right)$ is satisfied. Then for every real number $L$ with $L c>0$ and $|c| / 2<|L|<|c|$ there exists a (nonoscillatory) solution $x$ of the differential equation $\left(\tilde{\mathrm{E}}_{0}, \delta\right)$, that is of the equation

$\left(\tilde{\mathrm{E}}_{0}, \delta\right)$

$$
x^{(n)}(t)+\delta F(t ; x\langle g(t)\rangle)=0
$$

such that

$$
\lim _{t \rightarrow \infty} x^{(m)}(t)=L / m !
$$

and, if $m<n-1$,

$$
\lim _{t \rightarrow \infty} x^{(j)}(t)=0 \quad(j=m+1, \ldots, n-1) .
$$


COROLlaRY 3.4. Let $m, 0 \leqslant m \leqslant n-1$, be an integer such that the following condition is satisfied:

$\left(\tilde{\mathrm{C}}_{5}\right)$ For every nonzero constant $c$,

$$
\int^{\infty} t^{n-1-m}\left|F\left(t ; c\left[g_{1}(t)\right]^{m}, c\left[g_{2}(t)\right]^{m}, \ldots, c\left[g_{N}(t)\right]^{m}\right)\right| d t=\infty .
$$

Then for every nonoscillatory solution $x$ of the differential equation $\left(\tilde{\mathrm{E}}_{0}, \delta\right)$ with $x(t)=O\left(t^{m}\right)$ as $t \rightarrow \infty$,

$$
\delta(-1)^{n+m} x(t) x^{(m)}(t) \leqslant 0 \text { for all large } t
$$

and

$$
\lim _{t \rightarrow \infty} x^{(j)}(t)=0 \quad \text { monotonically } \quad(j=m, m+1, \ldots, n-1)
$$

COROllary 3.5. Let $m, 0 \leqslant m \leqslant n-1$, be an integer. Then the condition $\left(\tilde{\mathbf{C}}_{3}^{\prime}\right)$ is a necessary and sufficient condition in order that the differential equation $\left(\tilde{\mathrm{E}}_{0}, \delta\right)$ have a (nonoscillatory) solution $x$ so that the $\lim _{t \rightarrow \infty} x^{(m)}(t)$ exists in $\mathbf{R}-\{0\}$.

In the case considered, that is the case where $r_{0}=r_{1}=\cdots=r_{n-1}=1$, the conditions $\left(\mathrm{C}_{2}\right)$ and $\left(\mathrm{C}_{2}^{\prime}\right)$ follow from $\left(\tilde{\mathrm{C}}_{2}\right)$ and $\left(\tilde{\mathrm{C}}_{2}^{\prime}\right)$ respectively. Furthermore, we have the formula

$$
\int_{u}^{\infty} \int_{v}^{\infty}(s-v)^{\mu} p(s) d s d v=\int_{u}^{\infty} \frac{(s-u)^{\mu+1}}{\mu+1} p(s) d s,
$$

where $p$ is a continuous nonnegative function on $[u, \infty)$ and $\mu$ a nonnegative integer. By this formula, it is a matter of elementary calculus to see that in the considered case for any integer $m, 0 \leqslant m \leqslant n-1$, the conditions $\left(\mathrm{C}_{3}\right)$ and $\left(\mathrm{C}_{3}^{\prime}\right)$ follow from $\left(\tilde{\mathrm{C}}_{3}\right)$ and $\left(\tilde{\mathrm{C}}_{3}^{\prime}\right)$ respectively as well as for any integer $m, 1 \leqslant m \leqslant n-1$, the condition $\left(\mathrm{C}_{5}\right)$ follows from $\left(\tilde{\mathrm{C}}_{5}\right)$.

\section{Discussion}

Let us consider, in particular, the ordinary differential equation

$$
x^{(n)}(t)+a(t) \Phi(x(t))=0
$$

and the linear equation associated with it

$$
x^{(n)}(t)+a(t) x(t)=0,
$$

where $a$ is of constant sign and continuous on the interval $\left[t_{0}, \infty\right)$ and $\Phi$ is defined at least on $\mathbf{R}-\{0\}$ and has the following sign property:

$$
y \neq 0 \Rightarrow y \Phi(y)>0 \text {. }
$$


If the function $\Phi$ is increasing and such that

$$
\max \left\{\limsup _{y \rightarrow \infty} \frac{\Phi(y)}{y}, \limsup _{y \rightarrow-\infty} \frac{\Phi(y)}{y}\right\}<\infty,
$$

then, as it follows from Corollary 3.5 , the condition

$$
\int^{\infty} t^{n-1}|a(t)| d t<\infty
$$

is a necessary and sufficient condition in order that for any integer $m, 0 \leqslant m \leqslant n$ -1 , the equation (D) have at least one (nonoscillatory) solution $x$ so that the $\lim _{t \rightarrow \infty} x(t) / t^{m}$ exists in $\mathbf{R}-\{0\}$.

In particular, under condition $(\mathrm{H})$, the differential equation (d) has $n$ (nonoscillatory) solutions $x_{i}(i=0,1, \ldots, n-1)$ on $\left[t_{0}, \infty\right)$ with $\lim _{t \rightarrow \infty} x_{i}(t) / t^{i} \in(0, \infty)$ $(i=0,1, \ldots, n-1)$, which obviously constitute a base of the space of the solutions of $(\mathrm{d})$. Thus, condition $(\mathrm{H})$ ensures the nonoscillation of all (nontrivial) solutions of the equation (d).

If the function $\Phi$ is increasing and such that

$$
\min \left\{\liminf _{y \rightarrow \infty} \frac{\Phi(y)}{y}, \liminf _{y \rightarrow-\infty} \frac{\Phi(y)}{y}\right\}>0
$$

and the differential equation (D) admits at least one (nonoscillatory) solution $x$ with $\lim _{t \rightarrow \infty} x(t) / t^{m} \in \mathbf{R}-\{0\}$ for some integer $m, 0 \leqslant m \leqslant n-1$, then, by Corollary 3.5 , the equation (D) has at least one (bounded nonoscillatory) solution $x$ so that the $\lim _{t \rightarrow \infty} x(t)$ exists in $\mathbf{R}-\{0\}$.

\section{References}

C. V. Coffman and J. S. W. Wong (1972), 'Oscillation and nonoscillation theorems for second order ordinary differential equations', Funkcional. Ekvac. 15, 119-130.

W. A. Coppel (1971). Disconjugacy, Lecture Notes in Mathematics 220 (Springer-Verlag).

M. K. Grammatikopoulos, Y. G. Sficas and V. A. Staikos (1978), 'Asymptotic and oscillatory criteria for retarded differential equations', J. Math. Anal. Appl. 63, 59l-605.

T. Kusano and H. Onose (1975), 'Nonoscillatory solutions of differential equations with retarded arguments', Bull. Fac. Sci. Ibaraki Univ. Ser. A 7, 1-11.

T. Kusano and H. Onose (1976a), 'Asymptotic behavior of nonoscillatory solutions of functional differential equations of arbitrary order', J. London Math. Soc. 14, 106-112.

T. Kusano and H. Onose (1976b), 'Nonoscillation theorems for differential equations with deviating argument', Pacific J. Math. 63, 185-192.

G. Ladas (1971), 'Oscillation and asymptotic behavior of solutions of differential equations with retarded argument', J. Differential Equations 10, 281-290.

P. Marušiak (1973), 'Note on the Ladas' paper on oscillation and asymptotic behavior of solutions of differential equations with retarded argument', J. Differential Equations 13, 150-156.

$\mathrm{H}$. Onose (1973), 'Oscillation and asymptotic behavior of solutions of retarded differential equations of arbitrary order', Hiroshima Math. J. 3, 333-360. 
Ch.G. Philos (1977), "An oscillatory and asymptotic classification of the solutions of differential equations with deviating arguments', Atti Accad. Naz. Lincei Rend. Cl. Sci. Fis. Mat. Natur. 63, 195-203.

Ch.G. Philos (1978a), 'Oscillatory and asymptotic behavior of the bounded solutions of differential equations with deviating arguments', Hiroshima Math. J. 8, 31-48.

Ch.G. Philos (1978b), 'Oscillatory and asymptotic behavior of all solutions of differential equations with deviating arguments', Proc. Roy. Soc. Edinburgh Sect. A 81, 195-210.

Ch. G. Philos and V. A. Staikos (1979), 'Quick oscillations with damping', Math. Nachr. 92, 153-160.

Ch. G. Philos and V. A. Staikos (1980), 'Non-slow oscillations with damping', Rev. Roum. Math. Pures Appl. 25, 1099-1110.

J. Schauder (1930), 'Der Fixpunktsatz in Functionalräumen', Studia Math. 2, 171-180.

V. A. Staikos (1976), Differential equations with deviating arguments-oscillation theory, unpublished.

V. A. Staikos and Ch.G. Philos (1977a), 'On the asymptotic behavior of nonoscillatory solutions of differential equations with deviating arguments', Hiroshima Math. J. 7, 9-31.

V. A. Staikos and Ch.G. Philos (1977b), 'Asymptotic properties of nonoscillatory solutions of differential equations with deviating argument', Pacific J. Math. 70, 221-242.

V. A. Staikos and Ch.G. Philos (1978), 'Nonoscillatory phenomena and damped oscillations', Nonlinear Anal. 2, 197-210.

V. A. Staikos and Y. G. Sficas (1972), 'Criteria for asymptotic and oscillatory character of functional differential equations of arbitrary order', Boll. Un. Mat. Ital. 6, 185-192.

W. F. Trench (1975), 'Oscillation properties of perturbed disconjugate equations', Proc. Amer. Math. Soc. 52, 147-155.

A. Tychonoff (1935), 'Ein Fixpunktsatz,' Math. Ann. 111, 767-776.

Department of Mathematics

University of Ioannina

Ioannina

Greece 